\title{
A review of the state-of-the-art of assistive technology for people with ASD in the workplace and in everyday life
}

\author{
Lina J. Wali ${ }^{1}$ and Filippo Sanfilippo ${ }^{2(凶)}[0000-0002-1437-8368]$ \\ 1 Dept. of Science and Industry systems, University of South-Eastern Norway (USN), \\ Post box 235, 3603 Kongsberg, Norway \\ 2 Dept. of Engineering Sciences, University of Agder (UiA), \\ Jon Lilletuns vei 9, 4879, Grimstad, Norway \\ filippo.sanfilippo@uia.no
}

\begin{abstract}
Autism, also known as autism spectrum disorder (ASD), is an incurable brain-based disorder that refers to a wide range of complex neurodevelopment disorders characterised by marked difficulties in communication and social skills, repetitive behaviour, highly focused interests and sensory sensitivity. Autism can present challenges for affected people at the work environment and in everyday life. The barrier for individuals with ASD increases further with changing environmental situations. Individuals with ASD have limited abilities to isolate their Five senses and often experience over- or under-sensitivity to sounds, touch, tastes, smells, light, colours or temperatures. In this perspective, individuals with autism may experience extraordinary challenges during a regular day for most people, especially in non-conductive crowded environments like workplaces.

This work presents a survey of the state-of-the-art implementation as well as research challenges of assisting technology for people with ASD in the workplace and in everyday life. An overview of relevant key technologies and methods is outlined by focusing both on the therapeutic perspective as well as on the technological viewpoint. The aim of this paper is to provide a better understanding of the design challenges and to identify important research directions in this increasingly important area.
\end{abstract}

Keywords: Autism - Assistive Technology $\cdot$ ASD.

\section{Introduction}

Once considered a rare disorder, Autism or autism spectrum disorder (ASD) is nowadays a major public health problem affecting about $1 \%$ of the world population [46]. Nevertheless, numerous medical research works regarding the cause, prevention, treatment and cure of ASD, the medical area is still very restricted to specific facilitation techniques and interventions [40]. As a result, there is a population of individuals with autism having a variety of ages, genders and individual needs. This variety mostly depend on different ways of facilitation in one 


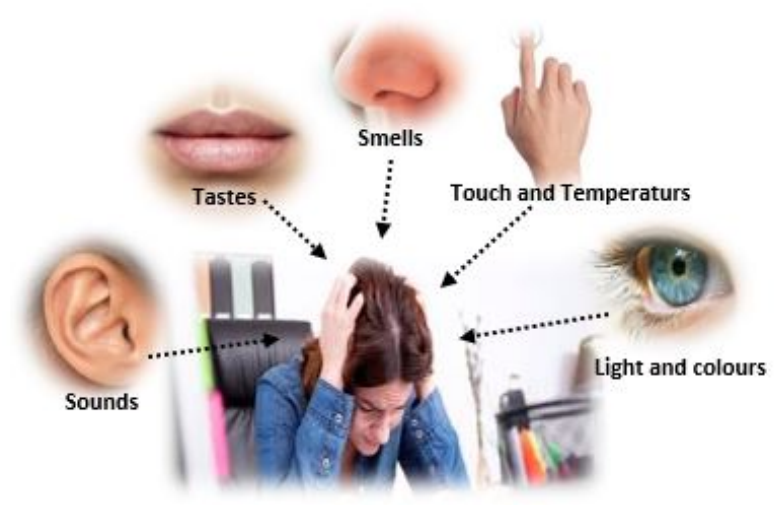

Fig. 1. The underlying idea of this work is to provide an overview of the state of the art in the area of assistive technology for people with Autism or autism spectrum disorder (ASD) in the workplace and in everyday life by considering different sensor channels, such as sounds, touch, tastes, smells, light, colours or temperatures.

or several areas throughout the individuals' lives. One of these areas is work or work environments where adults with ASD are facing daunting challenges. There is a good indication, that the challenges which individuals with autism face at work because of changing environmental situations are one of the causes of their remarkable unemployment rate of $80+\%$ [19]. Employment is an essential aspect of life for individuals including those with ASD [24]. "Work facilitates economic independence, engendering a sense of purpose and accomplishment, providing opportunities for socialisation and a mechanism through which to contribute to society" [41]. Hence, an unemployment rate of $80+\%$ of a group of people that currently constitutes $1 \%$ of the world population is very alarming. Nevertheless, in recent years, people with ASD have received improved attention from the labour market including some well-known employers such as Freddie Mac, Microsoft, SAP, Willis Towers Watson, Walgreens [23]. In parallel, there is a growing international interest in the use of assistive technology to support people with ASD and other impairments with life skills/independence [28].

Sensory sensitivity differences may be experienced by people with autism due to limited abilities to isolate their Five senses and often encounter over- or under-sensitivity to sounds, touch, tastes, smells, light, colours or temperatures. Therefore, individuals with autism may experience extraordinary challenges during a regular day for most people, especially in non-conductive crowded environments like workplaces. This paper aims at providing an overview of the state of the art in the area of assistive technology for people with ASD in the workplace and in everyday life by considering different sensor channels, as shown in Fig. 1. It can be extremely difficult to design technologies that fully engage the autistic user experience, especially when considering the impact of potential sensory 


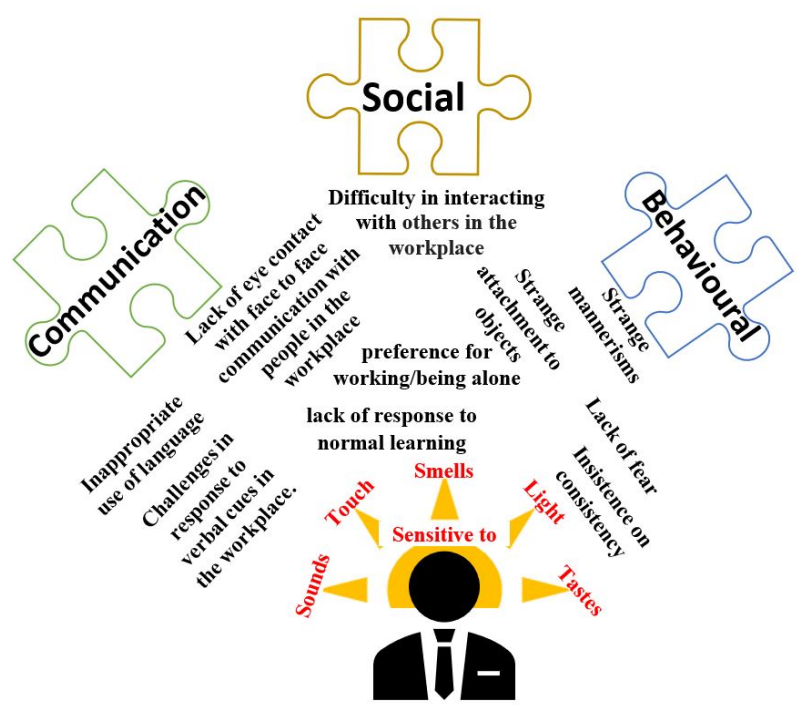

Fig. 2. Autism spectrum disorders are defined by social, communication and behavioural impairments and are associated with a range of symptoms [13].

impairments and multi-sensory integration. To support this kind of thoughtful assisting technologies, relevant key techniques and methods are presented by focusing both on the therapeutic perspective as well as on the technological viewpoint. On the basis of current trends, relevant design challenges are considered to identify the corresponding research directions in this increasingly important area.

The paper is organised as follows. A review of the related research work from a therapeutic perspective is given in Section 2. In Section 3, we focus on the description of the technological perspective. In Section 4, existing systems for obtaining an inclusive workplace are surveyed. A discussion is presented in Section 5. Finally, conclusions and future works are discussed in Section 6.

\section{Medical perspective}

\subsection{A brief background related to ASD}

Autism is a lifelong incurable brain-based disorder that refers to a group of complex neurodevelopment disorders resulting in a substantial burden for individuals, families and society [48]. It is primarily characterised by social-communication challenges and restricted repetitive behaviours, activities and interests [43], as shown in Fig. 2 [13].

Autism is caused by changes in the brain without knowing exactly why the changes occur. Researchers associate the development of autism to genetics and 
environmental factors such as infection during the pregnancy, premature birth, older age of parents and toxins in the environment [32]. However, the exact cause of autism is currently unknown, but still investigated. Different numbers are presented regarding the prevalence of ASD. According to Centers for Disease Control and Prevention (CDC)s' website, between 1\%-2\% of the world population in average has ASD [16]. Data show a steady rise of autism which has sparked fears of an autism epidemic. Only in the U.S., 1 in 59 children had a diagnosis of ASD by age 8 in 2014, a 15 percent increase over 2012 [16]. There are disagreements and different opinions and theories among researchers regarding the underlying cause of the more frequent occurrence of autism. According to numerous researches, the bulk of the increase stems from a growing awareness of autism and lower threshold for diagnosis, and expanded definition of ASD [47]. While according to other experts, the trend among parents in those past years of getting children in an older age is one of the causes. As the studies have shown older men and women are more likely than young ones to have a child with autism [31]. On the other hand, Dr. Stephanie Seneff, a senior research scientist at the Massachusetts Institute of Technology (MIT)'s Computer Science and Artificial Intelligence Laboratory, connects this rise to a particular toxic in the environment called Glyphosate and according to her by 2025, half the children born in the United States will be diagnosed with autism [3].

Autism occur in a spectrum, with a variety of different symptoms ranging from mild to severe, characteristics and level of intelligence quotient (IQ) as shown in Fig. 3 and Fig. 2. To help individuals with autism developing fundamental skills for good functioning throughout life, mental health professionals used to classify the autism spectrum into several different types. The primary types consisted of three groups as shown in Fig. 3:

- Asperger's syndrome. The individuals within this group, have a variety of symptoms milder than classical autism, specifically, in communication context and often tends to be normal or to above intelligent and able to handle their daily life;

- Pervasive developmental disorder not otherwise specified (PDD-NOS). The individuals within this group include the ones with more severe autism than Asperger's syndrome, but not as severe as autistic disorder;

- Autistic disorder or classical autism. This includes the same types of symptoms as Asperger's and PDD-NOS, but at a more intense level.

However, because of the confusion among professionals, family and teachers about the different types, it was concluded in 2013 to only use the term "Autism spectrum disorder" and herewith, based on their characteristics and impairments, individuals with ASD can be diagnosed as low, medium or high functioning. Regardless, it is not unusual to hear the former terms being used nowadays since the they are still widely adopted by professionals and other people in general.

The symptoms usually occur early and can be dedicated at 18 months or younger [26]. Hence, a reliable diagnosis can often be made at the age of 2 to 3. However, in many cases, the child does not receive a final diagnosis until 


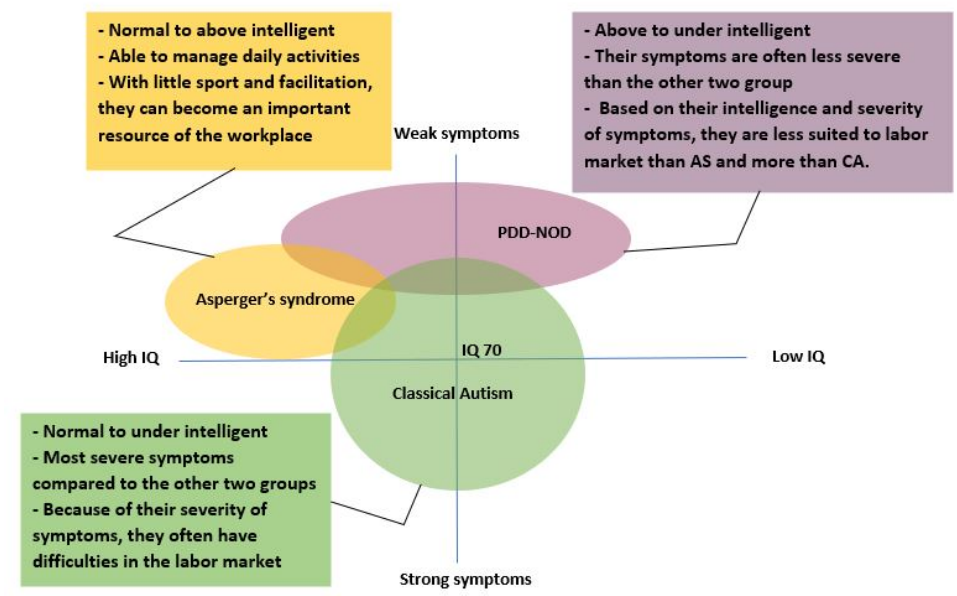

Fig. 3. The three sub-types of ASD vary in the severity of their symptoms and mental impairments [13].

later [2], [42]. The primary cause for this is the absence of medical or lab tests for diagnosing ASD [11]. So, instead of blood tests, brain scans or any other physical tests, the mental health professionals rather depend on observations of the child and feedback from the parents or carers [11]. Besides, the fact that ASD has an extensive range of symptoms makes the diagnostic process more complex, challenging and time consuming. This is unfortunate since "a growing body of evidence supports the value of early diagnosis and treatment with evidence-based interventions, which can significantly improve the quality of life of individuals with ASD as well as of their families. Particularly noteworthy are early interventions that occur in natural surroundings and can be modified to address age-related goals throughout the lifespan" [17].

\subsection{Challenges in everyday life and at the workplace for people with ASD}

People with autism often possess sought-after abilities and a desire to work. Also, they often have sought after valuable characteristics in the labour market such as mathematical acuity, exceptional computer skills, concentration skills, photographic and long-term memory, or tolerance for repetitive activities [21]. In addition, they tend to be known for being honest, reliable and displaying perseverance which can result productivity in the workplace. Even their impairment in social skills and communication, as shown in Fig. 2, can often lead to positive outcomes in the workplace by avoiding unnecessary social interactions [21]. Presently, there are numerous successful individuals with autism working in dif- 
ferent areas such as math, computer science and art [10]. This proves that many other people with ASD can achieve great achievments with the right support and guidance.

However, the main challenge is the large variation of ASD due to enormous individual differences and complexities of each individual [27], as shown in Fig. 3. It is widely accepted that the heterogeneity in the functional level within ASD is greater than in other clinical groups, and that the identication of severity and additional difculties is crucial to obtain personalised diagnoses. Addressing the challenges is therefore demanding and requires cutting edge expertise and collaboration between experts, local people and local authorities. Despite, the great variation of ASD, some of the symptoms are commonly observed among people with autism, some of which are in: cognitive and intellectual abilities, sensory processing, language and gestures, reciprocal conversation, movement and motor skills, eye contact, imagination, abstract and symbolic play, mental simulation, perception, affect and empathy, problem solving skills, executive functions, responses to sensory stimulation, adaptation to changes, generalization of learnt skills, anxiety management, cooperative working, managing phobias/fears such as loud noises, dogs, thunderstorm and vacuum cleaners, distinguishing between important and unimportant events/aspects [7]. As we can tell from the common symptoms, many of them are related to sensory challenges.

It is estimated that over $90+\%$ of people with ASD have sensory processing disorders (SPD) [8]. In fact, hyper- or hyporeactivity to sensory input or unusual interest in sensory aspects of the environment is now included in the Diagnostic and Statistical Manual of Mental Disorders, Fifth Edition (DSM-5)'s diagnostic criteria [8]. As a result, people with autism can be susceptible to changes and disadvantaged regarding employment [44]. For many people, habits, rituals and routines are a part of everyday life including their workday. People can become uneasy if the daily routine change while individuals with autism can have an unusual or more intense reaction. The case is the same or worse when it comes to environmental changing situations. A brighter light intensity, sound or smell in the background which most people would ignore can lead to anxiety or meltdown for people with autism. This is particularly demanding when it comes to crowded environments like workplaces where individuals with autism recognise stressful situations. By avoiding these problems, people with autism can have a better life quality and much more energy and willingness to do activities of daily living including better work productivity and performance. Fortunately, there are tools dealing with different aspects of the routine or habits challenges. However, assistive tools for reducing stress and increasing situational awareness are crucially needed but still missing [37].

\section{Technological perspective}

In existing literature, different works have been done to develop assistive technology interventions for people with ASD. However, they primarily focus on children, while only a few of them target adolescents or adults. Personal digital 
assistants (PDAs) are supported by emerging researches as assistive tools for cognition among people with various brain injuries such as intellectual disability, mental illness and Alzheimer's disease [21]. In recent years, this is motivated by the fact that PDAs such as smartphones and tablets have been developed into multi-functional devices that can incorporate advanced task managers. This makes PDAs a convenient tool for communication, a source for finding support, recording and editing videos and being a dynamic and growing source of information in academia [21]. These devices may be especially suitable for people with ASD since it is observed that people with ASD generally prefer instruction and support provided by computers rather than directly by another person [1]. Multiple studies support the use of PDAs for people with ASD at the workplace based on various strategies (e.g. time, task, task-training, social interactions and behavioural management) used in school and transitional settings [21].

Further, various studies confirm the challenges for people with ASD due to environmental changes and how they negatively affect their work performance and productivity. Some works suggest the idea that people with neurological conditions can be successfully supported by smart homes. This approach assumes that the needs and aspirations of people with ASD from the technological interventions are fully understood and integrated in the design [14]. However, the current approaches that can provide a customised work environment such as smart buildings or cities are both complex and expensive [22]. In contrast, there are some inexpensive assistive systems such as noise blocking, active noise cancelling (ANC), or noise reduction headphones [34]. These systems are widely used by people with ASD in the workplace and in everyday life. A brief description of each of them along with a comparison of advantages and disadvantages from the perspective of an individual with autism is depicted in ${ }^{3}$.

\section{Existing systems for an inclusive workplace}

Instead of isolating, an inclusive workplace is a work environment where the individuals are valued, integrated and included within their work force independently of their gender, background, religion and disability. Assistive technology can have a remarkable contribution to the inclusion of people with disability in the workplace. Thus, research in this area is essential for developing new assistive technologies for obtaining an inclusive workplace. In this perspective, supporting sensory needs in the workplace among people with ASD is of primary importance. There is a good body of literature and research that show how people with autism face challenges regarding sensory sensitivity [12], [33]. However, research about coping strategies and assistive technology used in the workplace is still limited.

Relevant studies related to different sensory channels are outlined in the following of this section. Challenges, missing gap and further proposals of different technological strategies that have potential for successful implementation in a

\footnotetext{
${ }^{3}$ https://medium.com/musings-from-mars/headphones-f1b64cfae7d1
} 
conductive workplace are grouped according to the different senses and presented. Finally, a review of the current promising assistive robotics technologies for interventions in an inclusive workplace is also outlined.

\subsection{Touch}

People with autism often tend to be sensitive to touch or close contact. This includes animals, food plants, textiles, other objects/materials and especially contact with other people. Thus, it is often easier for individuals with autism to relate to technological devices instead of direct contact with other people. In this context, the use of technological devices like computers, tablets and smart phones has been essential in many areas such as entertainment, education or school and therapeutically purposes. However, research on the use of this technology by workers with ASD is slight [21], [20]. According to the literature, this technology is primarily adopted for autistic children. A few studies show positive outcomes of using iPads and iPods as an assistive technology for autistic adults and adolescents [25], [29], [9]. A few works [21], [20] in this field focus on the use of a tablet as an assisive technology in the workplace. According to the work presented in [20], "Reducing the need for personal supports among workers with autism using an iPod touch as an assistive technology delayed randomised control trial". According to the same study, workers among the 50 autistic participants who were trained to use iPods in the beginning of their job required significant fewer hours of job coaching support. However, the participants in this study consisted of only high functioning adults with no intellectual, verbal communication or severe sensitive impairment.

\subsection{Eye sight}

individuals with autism often suffer from light sensitivity regardless of whether its source is natural or artificial. Sustained exposure to visual stimuli may cause pain, headache and in worst cases meltdown. Concentration challenges is also reported when exposed to fluorescent lighting or bright sunlight [6]. From a visual perspective, computerised smartglasses are being developed as an assistive technology for daily activities for children and adults with autism spectrum disorder (ASD) [36]. While smartglasses may be able to help with educational and behavioral needs, their usability and acceptability for children with ASD is largely unknown. Even less it is known when considering the workplace environment.

\subsection{Sound}

When considering sound, remote-microphone (RM) technology is employed to facilitate self-hearing [30] and distance communication [39]. The individual and group data from the last study suggest that RM technology may improve auditory function in children with ASD. However, no studies have been performed regarding the workplace environment to the best of the authors' knowledge. To 
complement this aspect, sensory headphones (sound blocking, active noise cancelling (ANC), or noise reduction headphones) are also adopted. Even though these devices are generally inexpensive, there are several drawbacks, such as feedback not available, no data shearing features, the possibility that individuals can feel overwhelmed while wearing them.

\subsection{Taste}

Regarding the sense of taste, only a few studies can be found in the literature. In [4], olfactory and taste functioning in individuals with autism were considered to characterise chemosensory processing. In general, this aspect may be also relevant when considering the workplace of people with ASD during breaks or lunches.

\subsection{Smell}

When considering smell, a mechanistic link between olfaction and ASD is proven in [35]. This aspect my be very relevant when considering specifc workplaces, such as food manufactures or chemical manufacturers.

\subsection{Multi-sensor channels}

The barriers to inclusion at the workplace for people with ASD may increase further with changing environmental situations. Deviations in multi-sensor channels may lead to increased stress. The intervention plans to instill positive behaviour support (PBS) suggest that a customised environment can minimise the impacts due to these variations. In this perspective, our research team previously proposed a novel framework which leverages the information from multi-sensor channels in a combined manner to customise the environment so that situational awareness (SA) can be improved [37]. The proposed framework allows for monitoring the environment by combining the information from different sensor channels including both personal sensors (i.e. on board of a mobile device) as well as environmental sensors/actuators (i.e. embedded in smart-buildings). In this preliminary work, a case study was considered through the development of a prototype for a mobile application and by reporting results on a scale model of a smart workplace with customisable environment.

\subsection{Robotics technologies for interventions in an inclusive workplace}

Robots have been shown to provoke proactive social behaviour in individuals with ASD, especially with children [18], [38], [15]. Robot therapy for autism has been explored as one of the first application domains in the field of socially assistive robotics (SAR), which aims at developing robots that assist people with special needs through social interactions [38]. Regarding multi-sensorial stimuli, 
an interactive robotic framework that delivers emotional and social behaviours for multi-sensory therapy for children with autism spectrum disorders was presented in [5]. The framework comprises emotion-based robotic gestures and facial expressions, as well as vision and audio-based monitoring system for quantitative measurement of the interaction. Special aspects of interacting with children with autism with multi-sensory stimuli and the potentials for personalised therapies for social and behavioural learning were considered. However, research on the use of this technology by workers with ASD is still slight. This technology has great potential, especially when considering to combine it with the previously mentioned technologies.

\section{Discussion}

The most common existing assistive technologies for people with ASD in the workplace can mainly be divided in to two groups. The first group include PDAs and is mostly used for the purpose of reducing the need of personal sport and being able to manage different tasks independently. PDAs are pocket-size, easily accessible and economical when compared to other solutions. There are numerous features and several apps [45] which can turn the device in to a personal assistive technology device based on their needs. However, the learning process of people with ASD when using them might be both time-consuming and costly for the employer. In addition, research about their affect for adolescents or adults in the workplace and in everyday life is limited [20].

On the other had, the second group includes a few approaches and solutions to deal with environmental changing situation to improve productivity of individuals with autism at the workplace and in everyday life. One of the most promising approach that could turn the office workplace into a customised conductive workplace is the development of smart building or cities, but this is both complex and expensive. The other solutions, such as the sensory headphones, are more economical and easy to develop from a technical point of view. However, they are only based on one sense (the hearing sense), provide no feedback data, have no data sharing features and therefore cannot provide a conductive work environment for people with ASD.

\section{Conclusion}

In this work, an overview of the state of the art of technological solutions for assisting people with autism spectrum disorder (ASD) in the day-to-day work was presented by considering different sensor channels. Relevant key technologies and methods were presented by focusing both on the therapeutic perspective as well as on the technological viewpoint. Relevant design challenges were considered to identify the corresponding research directions in this increasingly important area. When considering the current solutions and works in the area, there is a remarkable gap of research and there is little evidence to suggest that all individuals with ASD have access to appropriate assistive technologies. 
Assistive technology for people with ASD in the workplace and in everyday life is still at its infancy. However, there are strong results which can be used to build further upon from both a therapeutic perspective as well as on the technological viewpoint. One of the fundamental targets of this paper is to further increase efforts world-wide on realising the large variety of application possibilities offered by the current technology as a stepping-stone for new research and development within this field. This effort is also supported by our ongoing research [37].

\section{References}

1. Aliee, Z.S., Jomhari, N., Rezaei, R., Alias, N.: The effectiveness of managing split attention among autistic children using computer based intervention. Turkish Online Journal of Educational Technology-TOJET 12(2), 281-302 (2013)

2. Barbaro, J., Dissanayake, C.: Autism spectrum disorders in infancy and toddlerhood: a review of the evidence on early signs, early identification tools, and early diagnosis. Journal of Developmental \& Behavioral Pediatrics 30(5), 447-459 (2009)

3. Beecham, J.E., Seneff, S.: Is there a link between autism and glyphosate-formulated herbicides? Journal of Autism 3(1), 1 (2016)

4. Bennetto, L., Kuschner, E.S., Hyman, S.L.: Olfaction and taste processing in autism. Biological psychiatry 62(9), 1015-1021 (2007)

5. Bevill, R., Park, C.H., Kim, H.J., Lee, J.W., Rennie, A., Jeon, M., Howard, A.M.: Interactive robotic framework for multi-sensory therapy for children with autism spectrum disorder. In: Proc. of the 11th ACM/IEEE International Conference on Human-Robot Interaction (HRI). pp. 421-422 (2016)

6. Bogdashina, O.: Sensory perceptual issues in autism and asperger syndrome: different sensory experiences-different perceptual worlds. Jessica Kingsley Publishers (2016)

7. Bozgeyikli, L., Raij, A., Katkoori, S., Alqasemi, R.: A survey on virtual reality for individuals with autism spectrum disorder: Design considerations. IEEE Transactions on Learning Technologies 11(2), 133-151 (2018)

8. Chang, Y.S., Owen, J.P., Desai, S.S., Hill, S.S., Arnett, A.B., Harris, J., Marco, E.J., Mukherjee, P.: Autism and sensory processing disorders: shared white matter disruption in sensory pathways but divergent connectivity in social-emotional pathways. PloS one 9(7), e103038 (2014)

9. Coffin, A.B., Myles, B.S., Rogers, J., Szakacs, W.: Supporting the writing skills of individuals with autism spectrum disorder through assistive technologies. In: Technology and the Treatment of Children with Autism Spectrum Disorder, pp. 59-73. Springer (2016)

10. Connect, L.: 20 incredibly successful people on the autism spectrum, https://www. abadegreeprograms.net/successful-people-on-the-autism-spectrum/, last accessed on 2018-10-17

11. Connect, L.: Screening \& diagnosis, https:https://www.cdc.gov/ncbddd/autism/ screening.html, last accessed on 2018-10-17

12. Corbett, B.A., Schupp, C.W., Levine, S., Mendoza, S.: Comparing cortisol, stress, and sensory sensitivity in children with autism. Autism Research 2(1), 39-49 (2009)

13. David Lichtenstein: Behind the autism spectrum (2014), https://www . scienceinschool.org/2012/issue24/autism 
14. Dewsbury, G., Linskell, J.: Smart home technology for safety and functional independence: the uk experience. NeuroRehabilitation 28(3), 249-260 (2011)

15. Dickstein-Fischer, L.A., Crone-Todd, D.E., Chapman, I.M., Fathima, A.T., Fischer, G.S.: Socially assistive robots: current status and future prospects for autism interventions. Innovation and Entrepreneurship in Health 5, 15 (2018)

16. for Disease control, C., prevention: Data \& statistics (2019), https://www.cdc.gov/ ncbddd/autism/data.html, last accessed on 2018-10-17

17. Elder, J.H., Kreider, C.M., Brasher, S.N., Ansell, M.: Clinical impact of early diagnosis of autism on the prognosis and parent-child relationships. Psychology research and behavior management 10, 283 (2017)

18. Feil-Seifer, D., Matarić, M.J.: Toward socially assistive robotics for augmenting interventions for children with autism spectrum disorders. In: Experimental robotics. pp. 201-210. Springer (2009)

19. Frank, F., Jablotschkin, M., Arthen, T., Riedel, A., Fangmeier, T., Hölzel, L.P., van Elst, L.T.: Education and employment status of adults with autism spectrum disorders-a cross-sectional-survey. BMC psychiatry 18(1), 75 (2018)

20. Gentry, T., Kriner, R., Sima, A., McDonough, J., Wehman, P.: Reducing the need for personal supports among workers with autism using an ipod touch as an assistive technology: delayed randomized control trial. Journal of autism and developmental disorders 45(3), 669-684 (2015)

21. Gentry, T., Lau, S., Molinelli, A., Fallen, A., Kriner, R.: The apple ipod touch as a vocational support aid for adults with autism: Three case studies. Journal of Vocational Rehabilitation 37(2), 75-85 (2012)

22. Hancke, G.P., Hancke Jr, G.P., et al.: The role of advanced sensing in smart cities. Sensors 13(1), 393-425 (2012)

23. Heather Nezich: More companies hiring employees with autism spectrum disorder (2017), http://www.who.int/mediacentre/factsheets/fs282/fr/, last accessed on 2018-10-25

24. Hendricks, D.: Employment and adults with autism spectrum disorders: Challenges and strategies for success. Journal of Vocational Rehabilitation 32(2), 125-134 (2010)

25. Hillier, A., Greher, G., Queenan, A., Marshall, S., Kopec, J.: Music, technology and adolescents with autism spectrum disorders: The effectiveness of the touch screen interface. Music Education Research 18(3), 269-282 (2016)

26. Johnson, C.P., Myers, S.M., et al.: Identification and evaluation of children with autism spectrum disorders. Pediatrics 120(5), 1183-1215 (2007)

27. Lombroso, P.J., Ogren, M.P., Jones, W., Klin, A.: Heterogeneity and homogeneity across the autism spectrum: the role of development. Journal of the American Academy of Child \& Adolescent Psychiatry 48(5), 471-473 (2009)

28. Mintz, J., Gyori, M., Aagaard, M.: Touching the Future Technology for Autism?: Lessons from the HANDS Project, vol. 15. IOS Press (2012)

29. Nepo, K., Tincani, M., Axelrod, S., Meszaros, L.: ipod touch® to increase functional communication of adults with autism spectrum disorder and significant intellectual disability. Focus on Autism and Other Developmental Disabilities 32(3), 209-217 (2017)

30. Ni Chuileann, S.J., Quigley, J.: Recognizing voice: the child with autism spectrum disorder. Journal of Assistive Technologies 10(3), 140-152 (2016)

31. Parner, E.T., Baron-Cohen, S., Lauritsen, M.B., Jørgensen, M., Schieve, L.A., Yeargin-Allsopp, M., Obel, C.: Parental age and autism spectrum disorders. Annals of epidemiology 22(3), 143-150 (2012) 
32. Ratajczak, H.V.: Theoretical aspects of autism: Causesa review. Journal of immunotoxicology 8(1), 68-79 (2011)

33. Robertson, A.E., Simmons, D.R.: The relationship between sensory sensitivity and autistic traits in the general population. Journal of Autism and Developmental disorders 43(4), 775-784 (2013)

34. Rowe, C., Candler, C., Neville, M.: Noise reduction headphones and autism: A single case study. Journal of Occupational Therapy, Schools, \& Early Intervention 4(3-4), 229-235 (2011)

35. Rozenkrantz, L., Zachor, D., Heller, I., Plotkin, A., Weissbrod, A., Snitz, K., Secundo, L., Sobel, N.: A mechanistic link between olfaction and autism spectrum disorder. Current biology 25(14), 1904-1910 (2015)

36. Sahin, N.T., Keshav, N.U., Salisbury, J.P., Vahabzadeh, A.: Second version of google glass as a wearable socio-affective aid: Positive school desirability, high usability, and theoretical framework in a sample of children with autism. JMIR human factors $\mathbf{5}(1)$, e1 (2018)

37. Sanfilippo, F., Raja, K.: A multi-sensor system for enhancing situational awareness and stress management for people with asd in the workplace and in everyday life. In: Proceedings of the 52nd Hawaii International Conference on System Sciences (HICSS 2019), Maui, Hawaii, United States of America. pp. 4079-4086 (2019)

38. Scassellati, B., Admoni, H., Matarić, M.: Robots for use in autism research. Annual review of biomedical engineering 14, 275-294 (2012)

39. Schafer, E.C., Wright, S., Anderson, C., Jones, J., Pitts, K., Bryant, D., Watson, M., Box, J., Neve, M., Mathews, L., et al.: Assistive technology evaluations: Remote-microphone technology for children with autism spectrum disorder. Journal of communication disorders 64, 1-17 (2016)

40. Schall, C.M.: Positive behavior support: Supporting adults with autism spectrum disorders in the workplace. Journal of Vocational Rehabilitation 32(2), 109-115 (2010)

41. Scott, M., Falkmer, M., Falkmer, T., Girdler, S.: Evaluating the effectiveness of an autism-specific workplace tool for employers: A randomised controlled trial. Journal of autism and developmental disorders pp. 1-16 (2018)

42. Shattuck, P.T., Durkin, M., Maenner, M., Newschaffer, C., Mandell, D.S., Wiggins, L., Lee, L.C., Rice, C., Giarelli, E., Kirby, R., et al.: Timing of identification among children with an autism spectrum disorder: findings from a populationbased surveillance study. Journal of the American Academy of Child \& Adolescent Psychiatry 48(5), 474-483 (2009)

43. Texas Health and Human Services Commission: What is autism spectrum disorder? (2016), https://hhs.texas.gov/services/disability/autism/what-autism-spectrumdisorder, last accessed on 2018-10-19

44. Tomczak, M., Wójcikowski, M., Listewnik, P., Pankiewicz, B., Majchrowicz, D., Jdrzejewska-Szczerska, M.: Support for employees with asd in the workplace using a bluetooth skin resistance sensor-a preliminary study. Sensors 18(10), 3530 (2018)

45. of Western Australia, A.A.: Autismapps, https://www.autismapps.org.au/, last accessed on 2018-10-17

46. World Health Organisation: Facts and statistics (2014), http://www.who.int/ mediacentre/factsheets/fs282/fr/, last accessed on 2018-10-30

47. WRIGHT, J.: The real reasons autism rates are up in the u.s. (2017), https://www. scientificamerican.com/article/the-real-reasons-autism-rates-are-up-in-the- $\mathrm{u}-\mathrm{s} /$, last accessed on 2018-10-17

48. Xu, G., Strathearn, L., Liu, B., Bao, W.: Prevalence of autism spectrum disorder among us children and adolescents, 2014-2016. Jama 319(1), 81-82 (2018) 